\title{
AVALIAÇÃO DE HERBICIDAS NO CONTROLE DE PLANTAS DANINHAS EM MANDIOQUINHA-SALSA $\left({ }^{1}\right)$
}

\author{
MARIA APARECIDA NOGUEIRA SEDIYAMA $\left(2^{*}\right)$; ROGÉRIO SOARES DE FREITAS $\left({ }^{3}\right)$ \\ PEDRO CARLOS PEREIRA $\left({ }^{4}\right)$; TOCIO SEDIYAMA $\left({ }^{5}\right)$; MARIA HELENA TABIM MASCARENHAS $\left({ }^{2}\right)$; \\ FRANCISCO AFFONSO FERREIRA $\left({ }^{5}\right)$
}

\begin{abstract}
RESUMO
Avaliou-se a eficiência de herbicidas aplicados em pré e pós-emergência no controle de plantas daninhas e na produção de mandioquinha-salsa na Fazenda Experimental da EPAMIG, em Oratórios (MG), de maio a dezembro de 2002. Utilizou-se o delineamento de blocos casualizados com quatro repetições e 15 tratamentos incluindo duas testemunhas, com e sem capina. Em cada tratamento, foi usado um herbicida em pré e outro em pós-emergência das plantas, aplicados aos três e aos 45 dias após o plantio (DAP) respectivamente. A eficiência de controle e a toxidez dos herbicidas foram avaliadas aos $60 \mathrm{DAP}$ e a produção de matéria seca de plantas daninhas aos 60 e 130 DAP. Na colheita, avaliou-se produção de raízes, coroa e parte aérea. A emergência de plantas foi máxima aos $45 \mathrm{DAP}$ e, posteriormente, houve redução no estande, quando se utilizaram os herbicidas atrazine + óleo mineral e oxadiazon, em pós-emergência. Esses herbicidas, apesar de eficientes no controle de plantas daninhas, causaram toxidez à cultura. Na produção de raízes, destacaram-se os tratamentos: atrazine +fluazifop-p-butil; diuron + fluazifop-p-butil; diuron + Oxadiazon; linuron + fluazifop-p-butil; metolachlor + metribuzin + linuron e oxadiazon + diuron, com bom controle de plantas daninhas e rendimentos de raízes comerciais semelhantes ao da testemunha mantida no limpo $\left(8,62 \mathrm{t} \mathrm{ha}^{-1}\right)$.
\end{abstract}

Palavras-chave: Arracacia xanthorrhiza, controle químico, toxidez, seletividade.

\section{ABSTRACT \\ HERBICIDE EFFICIENCY IN CONTROLLING WEEDS IN PERUVIAN CARROT}

The efficiency of herbicides applied in pre- and post-emergence for weed control in Peruvian carrot production was evaluated at the EPAMIG Experimental Farm, Oratórios, Minas Gerais State, from May to December, 2002. The experiment was arranged in a randomized block design, with four replications and 15 treatments, including two controls (with and without weeding). Pre- and post-emergence herbicides were used in each treatment applied at 3 and 45 days after planting (DAP), respectively. Efficiency and toxicity were evaluated at $60 \mathrm{DAP}$ and weed dry matter yield at 60 and $130 \mathrm{DAP}$. At the time of harvest, the yield of root, crown and aerial parts were evaluated. There were no differences among treatments for plant emergence. Plant emergence was highest at $45 \mathrm{DAP}$ and later, followed by a decrease in the stand when atrazine + mineral oil and oxadiazon were used in pre-emergence. Despite being efficient, these herbicides caused toxicity to the crop. The following treatments stood out for root yield: atrazine + fluazifop-p-butil, diuron + fluazifop-p-butil, diuron + oxadiazon, linuron + fluazifop-p-butil, metolachlor + metribuzin + linuron e oxadiazon + diuron, giving good weed control and yield of commercial roots similar to the control kept with no weed $\left(8.62 \mathrm{t} \mathrm{ha}{ }^{-1}\right)$.

Key words: Arracacia xanthorrhiza, chemical control, toxicity, selectivity.

( $\left.{ }^{1}\right)$ Recebido para publicação em 28 de agosto de 2007 e aceito em 14 de maio de 2008.

$\left({ }^{2}\right)$ EPAMIG, Caixa Postal 216, 36570-977 Viçosa (MG). E-mail: marians@epamig.ufv.br (*) Autora correspondente.

$\left({ }^{3}\right)$ APTA/Pólo Regional do Noroeste Paulista, Caixa Postal 61, 15500-970 Votuporanga (SP).

$\left({ }^{4}\right)$ Bolsista FAPEMIG/EPAMIG-CTZM, Caixa Postal 216, 36570-977 Viçosa (MG).

$\left({ }^{5}\right)$ Departamento de Fitotecnia, Universidade Federal de Viçosa, 36570-977 Viçosa (MG). 


\section{Introdução}

O Brasil é o maior produtor de mandioquinhasalsa, com área cultivada em torno de 13.000 ha, em 2000 (Henz, 2002). Os Estados do Paraná e de Minas Gerais são os principais produtores, cuja produtividade média é de 8,8 t.ha ${ }^{-1}$ (SAntos, 1997). Minas Gerais possui mais de 6.000 ha cultivados em mais de 100 municípios e a Região Sul do Estado é a mais expressiva, correspondendo a, aproximadamente, $70 \%$ da produção (SANTOS, 1997; SANTOS et al., 2000).

Apesar de ser uma cultura bastante rústica e cultivada com baixos níveis de insumos externos como fertilizantes e defensivos, a interferência das plantas daninhas na cultura causa redução no rendimento de raízes comerciais superior a $98 \%$, quando em convivência por todo o ciclo. O período crítico de prevenção à interferência está entre 58 e 120 dias após o plantio (Freitas et al., 2004a). Nessa condição, a sobrevivência das plantas de mandioquinha-salsa é de apenas 66\% (Mascarenhas e Pereira, 1997).

A crescente escassez e valorização da mão-deobra rural tornam os herbicidas seletivos e eficientes para o controle de plantas daninhas uma ferramenta indispensável para o cultivo da mandioquinha-salsa (MASCAREnHAs, 1984).

O controle mecânico de plantas daninhas, com uso de enxada é eficiente, mas muito oneroso, consome parte expressiva da mão-de-obra da propriedade e, em épocas de chuvas, há baixa eficiência, aumentando o número de capinas e, conseqüentemente, a demanda de mão-de-obra, o que resulta no aumento do custo de produção dessa hortaliça. Por esse motivo, é importante a escolha de herbicidas seletivos à cultura e eficientes no controle das infestantes com grande potencial de uso para aqueles agricultores que querem permanecer na atividade. Freitas et al. (2004b) avaliaram e constataram herbicidas seletivos e com potencial de uso na cultura de mandioquinha-salsa. Para o controle químico de gramíneas anuais e algumas perenes, o fluazifopp-butil é eficiente (MASCARENHAS, 1984; Freitas et al., 2004b) e, em outras situações, o metribuzin controla espécies de folhas largas e algumas gramíneas, todavia ainda não existem herbicidas registrados no Ministério da Agricultura, Pecuária e Abastecimento para a cultura da mandioquinhasalsa (Rodrigues e Almeida, 2005). Objetivou-se avaliar o efeito de herbicidas aplicados em pré e em pós-emergência no controle de espécies daninhas e na produção de raízes comerciais de mandioquinha-salsa.

\section{Material e Métodos}

O experimento foi realizado na Fazenda Experimental da Empresa de Pesquisa Agropecuária de Minas Gerais (EPAMIG), no município de Oratórios (MG), no período de $3 / 5$ a 9/12/2002. O solo utilizado, Argissolo Vermelho-Amarelo câmbico, fase terraço, textura argilosa, possui, na camada de 0 a $20 \mathrm{~cm}$, as seguintes características: $\mathrm{pH}$ (água 1:2,5) $=5,98$; matéria orgânica $=2,34 \mathrm{dag}$ $\mathrm{kg}^{-1} ; \mathrm{P}=4,0 \mathrm{mg} \mathrm{dm}^{-3} ; \mathrm{K}=115 \mathrm{mg} \mathrm{dm}^{-3} ; \mathrm{Ca}^{2+}=2,67$ $\mathrm{cmol}_{\mathrm{c}} \mathrm{dm}^{-3} ; \mathrm{Mg}^{2+}=1,01 \mathrm{cmol}_{\mathrm{c}} \mathrm{dm}^{-3} ; \mathrm{Al}^{3+}=0,0 \mathrm{cmol}_{\mathrm{c}}$ $\mathrm{dm}^{-3} ; \mathrm{H}+\mathrm{Al}=1,3 \mathrm{cmol}_{\mathrm{c}} \mathrm{dm}^{-3} ; \mathrm{SB}=3,98 \mathrm{cmol}_{\mathrm{c}} \mathrm{dm}^{-3}$; $\mathrm{CTC}(\mathrm{t})=3,98 \mathrm{cmol}_{\mathrm{c}} \mathrm{dm}^{-3} ; \mathrm{CTC}(\mathrm{T})=5,32 \mathrm{cmol}_{\mathrm{c}} \mathrm{dm}^{-3}$; $\mathrm{V}=75 \%$ e $\mathrm{m}=0,0 \%$. Os dados das temperaturas máxima e mínima, da umidade relativa do ar e da precipitação pluvial são apresentados na tabela 1 .

Tabela 1. Médias mensais das temperaturas máxima (TM) e mínima (Tm), da umidade relativa do ar (UR) e da precipitação pluvial total mensal (PPT) durante o período de condução do experimento, em Oratórios (MG), 2002

\begin{tabular}{|c|c|c|c|c|}
\hline Meses & TM & $\operatorname{Tm}\left({ }^{\circ} \mathrm{C}\right)$ & UR & PPT \\
\hline & 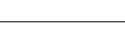 & 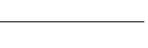 & $\%$ & $\mathrm{~mm}$ \\
\hline Maio & 27,61 * & 14,64 & 69,87 & 48,75 \\
\hline Junho & 27,33 & 11,62 & 69,70 & 1,26 \\
\hline Julho & 26,26 & 12,88 & 70,29 & 1,76 \\
\hline Agosto & 28,16 & 11,96 & 69,58 & 2,39 \\
\hline Setembro & 28,47 & 13,40 & 74,30 & 103,77 \\
\hline Outubro & 27,69 & 16,32 & 62,65 & 26,04 \\
\hline Novembro & 25,95 & 18,34 & 75,29 & 251,32 \\
\hline Dezembro & 27,14 & 19,93 & 78,65 & 277,11 \\
\hline
\end{tabular}

*Dados fornecidos pelo Departamento de Engenharia Agrícola - Centro de Ciências Agrárias - Universidade Federal de Viçosa (UFV), Viçosa (MG). 
O preparo do solo consistiu de aração, gradagem e levantamento das leiras com $30 \mathrm{~cm}$ de altura. A adubação de plantio foi feita com $180 \mathrm{~kg} \mathrm{ha}^{-1}$ de $\mathrm{P}_{2} \mathrm{O}_{5}$ na forma de superfosfato simples, aplicado no sulco de plantio, de acordo com CASALI (1999).

Utilizou-se o delineamento de blocos casualizados com quatro repetições e 15 tratamentos, correspondendo à combinação de herbicidas em pré e em pós-emergência, incluindo duas testemunhas, uma sem e outra com capina a enxada durante o ciclo (Tabela 2). Cada parcela foi constituída por três fileiras de plantas, com $6 \mathrm{~m}$ de comprimento, com 45 plantas/parcela, no espaçamento de 0,40 × 0,90 m. No plantio, foram utilizadas mudas de mandioquinhasalsa, clone Amarelo, com 12 meses de ciclo, preparadas de acordo com as recomendações para a cultura (SEDIYAma e CASAli, 1997).

Os tratamentos foram definidos de acordo com estudos prévios de seletividade, tolerância da cultura a herbicidas em pré e pós emergência e período de interferência com plantas daninhas (Pereira et al., 2003; FreitAs et al., 2004a, b).

Os herbicidas usados em pré-emergência foram aplicados aos três dias após o plantio (DAP) e aqueles em pós-emergência, aos $45 \mathrm{DAP}$, utilizando pulverizador costal pressurizado a $\mathrm{CO}_{2}$ a $2,4 \mathrm{kgf.cm}{ }^{-2}$, equipado com barra com quatro pontas de jato plano - tipo "leque" 110.03, proporcionando um volume de calda de 200 L.ha" ${ }^{1}$. As irrigações foram feitas por aspersão.

Tabela 2. Tratamentos, nome técnico e modo de aplicação, doses do ingrediente ativo (i.a.) e porcentagem de emergência de plantas de mandioquinha-salsa, em diferentes épocas de avaliação, em Oratórios (MG), 2002

\begin{tabular}{|c|c|c|c|c|c|c|}
\hline \multirow{3}{*}{ Tratamentos } & \multicolumn{2}{|c|}{ Nome técnico } & \multirow{3}{*}{ Dose do i.a. } & \multirow{2}{*}{\multicolumn{3}{|c|}{$\begin{array}{c}\text { Emergência de plantas } \\
\text { (Dias após o plantio) }\end{array}$}} \\
\hline & \multicolumn{2}{|c|}{ Modo de aplicação } & & & & \\
\hline & \multirow[t]{2}{*}{ Pré-emergência } & \multirow[t]{2}{*}{ Pós-emergência } & & 30 & 45 & 60 \\
\hline & & & kg ou L ha ${ }^{1}$ & \multicolumn{3}{|c|}{$\longrightarrow$ dias } \\
\hline 1 & Atrazine & Atrazine + óleo mineral 300g/L & $2,75+1,20$ & $75,00 \mathrm{a}$ & 93,89 a & $80,00 \mathrm{~b}$ \\
\hline 2 & Atrazine & Fluazifop-p-butil & $2,75+0,19$ & $76,67 \mathrm{a}$ & 90,56 a & $90,00 \mathrm{a}$ \\
\hline 3 & Atrazine & Oxadiazon & $2,75+0,75$ & $73,89 \mathrm{a}$ & $86,11 \mathrm{a}$ & $78,89 \mathrm{~b}$ \\
\hline 4 & Diuron & Fluazifop-p-butil & $1,60+0,19$ & $67,22 \mathrm{a}$ & 86,67 a & 88,89 a \\
\hline 5 & Diuron & Oxadiazon & $1,60+0,75$ & $70,56 \mathrm{a}$ & 89,44 a & $85,56 \mathrm{~b}$ \\
\hline 6 & Linuron & Fluazifop-p-butil & $1,48+0,19$ & $85,00 \mathrm{a}$ & 99,44 a & $99,44 \mathrm{a}$ \\
\hline 7 & Metolachlor/metribuzin ${ }^{1}$ & Fluazitop-p-butil & $2,88+0,19$ & 81,67 a & $94,44 \mathrm{a}$ & $95,55 \mathrm{a}$ \\
\hline 8 & Metolachlor/metribuzin ${ }^{1}$ & Linuron & $2,88+1,35$ & $74,44 \mathrm{a}$ & $92,22 \mathrm{a}$ & $94,44 \mathrm{a}$ \\
\hline 9 & Metolachlor/metribuzin ${ }^{1}$ & Oxadiazon & $2,88+0,75$ & $58,89 \mathrm{a}$ & $90,00 \mathrm{a}$ & $92,22 \mathrm{a}$ \\
\hline 10 & Metolachlor/metribuzin ${ }^{1}$ & Diuron & $2,88+1,25$ & 67,78 a & $92,22 \mathrm{a}$ & 91,67 a \\
\hline 11 & Metolachlor/metribuzin ${ }^{1}$ & Ioxynil & $2,88+0,50$ & $83,33 \mathrm{a}$ & $95,00 \mathrm{a}$ & $91,67 \mathrm{a}$ \\
\hline 12 & Oxadiazon & Fluazifop-p-butil & $1,00+0,19$ & $72,78 \mathrm{a}$ & $91,11 \mathrm{a}$ & $95,00 \mathrm{a}$ \\
\hline 13 & Oxadiazon & Diuron & $1,00+1,25$ & $78,33 \mathrm{a}$ & $91,11 \mathrm{a}$ & $88,88 \mathrm{a}$ \\
\hline 14 & \multicolumn{2}{|c|}{ Testemunha c/ capina } & - & $69,44 \mathrm{a}$ & 93,89 a & $93,33 \mathrm{a}$ \\
\hline 15 & \multicolumn{2}{|c|}{ Testemunha s/ capina } & - & $75,00 \mathrm{a}$ & 89,44 a & $89,44 \mathrm{a}$ \\
\hline CV (\%) & & - & - & 16,10 & 7,54 & 7,45 \\
\hline
\end{tabular}

Grupos de médias seguidas da mesma letra, na coluna, não diferem pelo teste Scott-Knott a $5 \%$ de probabilidade.

$\left.{ }^{1}\right)$ Metolachlor + metribuzin foi utilizado na dose $2,52+0,36 \mathrm{~kg}$ i.a. ha ${ }^{-1}$ respectivamente.

Aos 30, 45 e 60 DAP foram feitas avaliações da emergência de plantas de mandioquinha-salsa. A avaliação da toxidez dos herbicidas foi realizada aos 60 DAP utilizando-se a escala de 0 a 100 , onde zero é ausência de sintomas e 100, a morte das plantas.

A avaliação da eficácia dos herbicidas foi estimada por notas de porcentagem de controle aos 60 DAP e por amostragens das espécies daninhas predominantes aos 60 e $130 \mathrm{DAP}$. As espécies daninhas delimitadas por um gabarito de ferro de 0,50 $x$ 0,50 m foram cortadas rente ao solo, identificadas e pesadas. Em seguida, foram colocadas em sacos de papel e levadas para estufa com circulação de ar, a $70+2{ }^{\circ} \mathrm{C}$ por 72 horas, determinando a biomassa seca. As espécies daninhas predominantes na área experimental foram: Cyperus rotundus (tiririca), Siegesbeckia orientalis (botão-de-ouro), Brachiaria plantaginea (capim-marmelada), Brachiaria decumbens (capim-braquiária), Ipomoea grandifolia (corda-deviola), Bidens pilosa (picão-preto), Amaranthus spp. (caruru) e Ageratum conyzoides (mentrasto). 
A colheita foi feita aos 210 DAP, quando se avaliaram a produção de raízes comercial, não comercial e total, a biomassa fresca da parte aérea e de coroa. Os resultados foram submetidos à análise de variância e as médias agrupadas pelo teste de Skott-Knott, a 5\% de probabilidade.

\section{Resultados e Discussão}

A emergência de plantas de mandioquinhasalsa foi máxima aos $45 \mathrm{DAP}$, não havendo diferença entre os tratamentos. CÂMARA (1984) obteve a máxima emergência de plantas de mandioquinha-salsa aos 30 DAP. Até os 45 DAP, nenhum dos herbicidas aplicados em pré-emergência afetou o estande da cultura e nem intoxicou as plantas. Entretanto, aos 15 dias após aplicação dos herbicidas em pósemergência, ou seja, aos $60 \mathrm{DAP}$, verificou-se redução no número de plantas emergidas nos tratamentos 1 , 3 e 5 (Tabela 2).

A aplicação seqüencial que incluiu atrazine + óleo mineral, oxadiazon, diuron e ioxynil, ou seja, tratamentos 1, 3, 5, 9, 10, 11 e 13 foram os de maiores níveis de toxidez (Figura 1). Segundo Pereira et al. (2003) e Freitas et al. (2004b), o oxadiazon causa toxidez às plantas de mandioquinha-salsa, quando, em pós-emergência, até 28 dias após a sua aplicação e, dependendo da dose usada, observa-se boa recuperação das plantas.

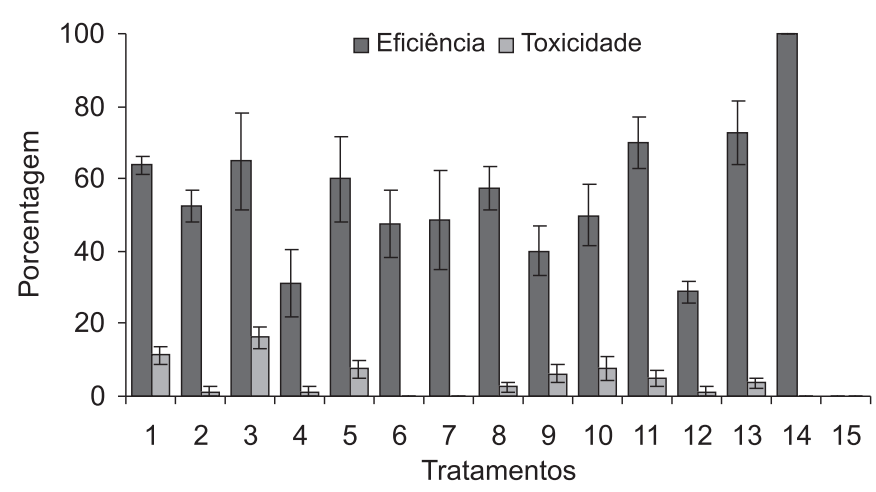

Figura 1. Eficiência e toxidez dos herbicidas aos 60 dias após o plantio da mandioquinha-salsa, considerando os diferentes tratamentos aplicados em pré e pósemergência (1 - Atrazine + Atrazine com óleo; 2 Atrazine + Fluazifop-p-butil; 3 - Atrazine + Oxadiazon; 4 - Diuron + Fluazifop-p-butil; 5 - Diuron + Oxadiazon; 6 - Linuron + Fluazifop-p-butil; 7 Metol.+Metr+Fluazifop-p-butil; 8 - Metolac.+Metrib+ Linuron; 9 - Metola.+Metrib+ Oxadiazon; 10 Metolac.+Metrib + Diuron; $11-$ Metolac. + Metrib + Ioxynil; 12 - Oxadiazon+Fluazifop-p-butil; 13 Oxadiazon + Diuron; 14 - Testemunha c/ capina; 15 Testemunha s/ capina), em Oratórios (MG), 2002.
Na primeira avaliação, aos 60 DAP (15 dias após aplicação dos herbicidas em pós-emergência), verificou-se, para a maioria dos tratamentos, mais de $50 \%$ de controle de plantas daninhas. A menor eficiência, ou seja, menor porcentagem de controle aos 60 DAP foi obtida nos tratamentos 4, 9 e $12 \mathrm{com}$ as combinações dos herbicidas diuron + fluazifop-pbutil, metolachlor + metribuzin + oxadiazon e oxadiazon + fluazifop-p-butil (Figura 1).

$\mathrm{Na}$ avaliação realizada aos 130 DAP, verificou-se que os tratamentos 1,3,7 e 12, ou seja, atrazine + atrazine + óleo mineral, atrazine + oxadiazon, metolachlor + metribuzin + fluazifop-pbutil e oxadiazon + fluazifop-p-butil respectivamente, tiveram a menor eficiência de controle, resultando em maior biomassa seca de plantas daninhas (Figura 2).

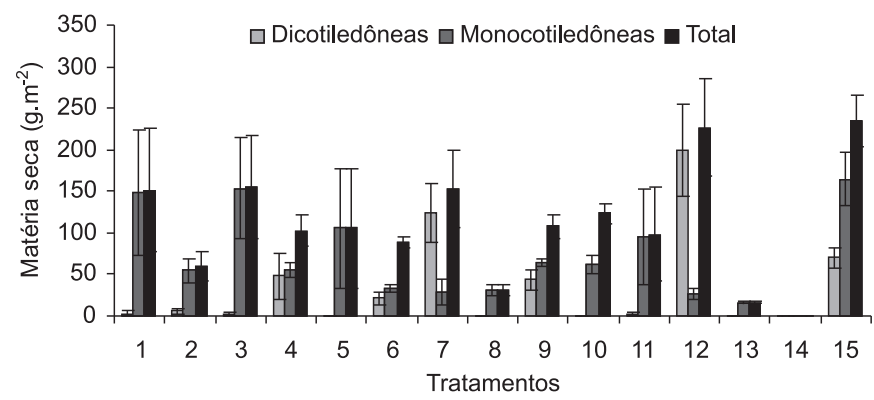

Figura 2. Matéria seca de plantas daninhas, aos 130 dias após o plantio de mandioquinha-salsa, considerando os diferentes tratamentos $(1$ - Atrazine + Atrazine com óleo; 2 - Atrazine + Fluazifop-p-butil; 3 - Atrazine + Oxadiazon; 4 - Diuron + Fluazifop-p-butil; 5 - Diuron + Oxadiazon; 6 - Linuron + Fluazifop-p-butil; 7 Metol.+Metr+Fluazifop-p-butil; 8 - Metolac.+Metrib+ Linuron; 9 - Metola.+Metrib+ Oxadiazon; 10 Metolac.+Metrib + Diuron; 11 - Metolac.+Metrib + Ioxynil; 12 - Oxadiazon+Fluazifop-p-butil; 13 Oxadiazon + Diuron; 14 - Testemunha c/ capina; 15 Testemunha s/ capina) aplicados em pré e pósemergência, em Oratórios (MG), 2002.

Em razão das condições climáticas da região não permitir o cultivo da mandioquinha-salsa durante o verão (Tabela 1), optou-se pela colheita precoce aos sete meses, enquanto o ciclo normal é 10 a 12 meses (Sediyama e Casali, 1997). Contudo, a produtividade alcançada foi semelhante à média nos principais Estados produtores (SANTOS, 1997).

A produção de biomassa fresca da parte aérea foi reduzida em todos os tratamentos com herbicidas quando comparadas à testemunha capinada. Por outro lado, a produtividade de raízes comerciais nos tratamentos 2, 4, 5, 6, 8 e 13 foi semelhante à da testemunha capinada, cujas plantas foram mantidas no limpo por todo o ciclo (Tabela 3). Pelos resultados, 
verifica-se que a planta de mandioquinha-salsa tolera certo nível de redução da parte aérea provocada pela interferência de plantas daninhas ou até mesmo causada por toxidez de herbicidas, sem afetar a produção de raízes comerciais. Este fato sugere que, além de sua rusticidade, a cultura também possui certo nível de plasticidade fisiológica. A plasticidade é a capacidade da planta em alterar sua morfologia e componentes de rendimento, a fim de adequá-los às condições impostas pelo arranjo de plantas (Cooperative Extension Service Ames, 1994).

O número de raízes e a produção de raízes não comerciais seguiram a mesma tendência da produção de raízes comerciais. Em alguns tratamentos que proporcionaram maior produção de raízes comerciais, também ocorreu maior produção de coroa (Tabela 3).

Os tratamentos 2, 4, 5, 6, 8 e 13 (Tabela 3) que proporcionaram produtividade semelhante à da testemunha mantida no limpo por todo o ciclo, também foram aqueles com os melhores níveis de controle de plantas daninhas mono e dicotiledôneas (Figuras 1 e 2). Contudo, para maior segurança, mais estudos devem ser realizados, especialmente para os tratamentos 2, 4, 6 e 8 por serem considerados promissores e pelo fato de ainda não existir herbicidas registrados para o controle de plantas daninhas em mandioquinha-salsa.

Alguns tratamentos propiciaram controle satisfatório das plantas daninhas durante o período crítico de sua interferência com a mandioquinha-salsa, que ocorre de 58 a 120 dias após o plantio (Freitas 2004a). Por outro lado, os tratamentos 9 e 11, mesmo com controle satisfatório (Figuras 1 e 2), proporcionaram menores produtividades que a da testemunha capinada (Tabela 3). Esse fato pode ter ocorrido pelo maior nível de toxidez às plantas de mandioquinha-salsa nos tratamentos já citados.

Tabela 3. Produção de mandioquinha-salsa, aos 210 dias após o plantio, considerando os diferentes tratamentos aplicados em pré e pós-emergência, em Oratórios (MG), 2002

\begin{tabular}{|c|c|c|c|c|c|}
\hline Tratamentos & $\begin{array}{l}\text { Parte } \\
\text { Aérea }\end{array}$ & $\begin{array}{c}\text { Raízes } \\
\text { Comerciais }\end{array}$ & $\begin{array}{l}\text { Raízes não } \\
\text { Comerciais }\end{array}$ & Coroa & $\begin{array}{c}\text { Raízes } \\
\text { Comerciais }\end{array}$ \\
\hline & \multicolumn{4}{|c|}{$-\mathrm{t} / \mathrm{ha}$} & n. ${ }^{\circ}$ \\
\hline 1 Atrazine + Atrazine com óleo & $4,14 \mathrm{c}$ & $2,41 \mathrm{c}$ & $1,31 \mathrm{c}$ & $0,23 \mathrm{c}$ & $31.250 \mathrm{c}$ \\
\hline 2 Atrazine + Fluazifop-p-butil & $9,59 \mathrm{~b}$ & 5,07 a & $1,75 \mathrm{~b}$ & $0,32 \mathrm{~b}$ & $55.469 \mathrm{~b}$ \\
\hline 3 Atrazine + Oxadiazon & $6,15 c$ & $3,34 \mathrm{~b}$ & $1,27 \mathrm{c}$ & $0,29 \mathrm{~b}$ & $39.063 \mathrm{~b}$ \\
\hline 4 Diuron + Fluazifop-p-butil & $9,30 \mathrm{~b}$ & $5,79 \mathrm{a}$ & $2,17 \mathrm{a}$ & $0,33 \mathrm{~b}$ & $58.594 \mathrm{~b}$ \\
\hline 5 Diuron + Oxadiazon & $9,63 \mathrm{~b}$ & $4,95 \mathrm{a}$ & $1,78 \mathrm{~b}$ & $0,36 \mathrm{~b}$ & $50.000 \mathrm{~b}$ \\
\hline 6 Linuron + Fluazifop-p-butil & $10,30 \mathrm{~b}$ & $7,23 \mathrm{a}$ & $2,12 \mathrm{a}$ & 0,49 a & 76.563 a \\
\hline 7 Metolachlor+Metr+Fluazifop-p-butil & $9,45 \mathrm{~b}$ & $4,22 \mathrm{~b}$ & $1,63 \mathrm{~b}$ & $0,34 \mathrm{~b}$ & $48.438 \mathrm{~b}$ \\
\hline 8 Metolachlor+Metrib + Linuron & $11,16 \mathrm{~b}$ & $6,13 \mathrm{a}$ & $1,65 \mathrm{~b}$ & $0,47 \mathrm{a}$ & $65.625 \mathrm{a}$ \\
\hline 9 Metolachlor.+Metrib + Oxadiazon & $8,80 \mathrm{~b}$ & $4,07 \mathrm{~b}$ & $1,20 \mathrm{c}$ & $0,27 \mathrm{~b}$ & $46.094 \mathrm{~b}$ \\
\hline 10 Metolachlor+Metrib + Diuron & $9,61 \mathrm{~b}$ & $4,27 \mathrm{~b}$ & $1,62 \mathrm{~b}$ & $0,30 \mathrm{~b}$ & $47.656 \mathrm{~b}$ \\
\hline 11 Metolachlor+Metrib + Ioxynil & $5,16 \mathrm{c}$ & $3,57 \mathrm{~b}$ & $1,42 \mathrm{~b}$ & 0,39 a & $51.563 \mathrm{~b}$ \\
\hline 12 Oxadiazon+Fluazifop-p-butil & $9,06 \mathrm{~b}$ & $4,67 \mathrm{~b}$ & $1,16 \mathrm{c}$ & $0,36 \mathrm{~b}$ & $50.000 \mathrm{~b}$ \\
\hline 13 Oxadiazon + Diuron & $13,12 \mathrm{~b}$ & 4,99 a & $1,53 \mathrm{~b}$ & $0,35 \mathrm{~b}$ & $53.906 \mathrm{~b}$ \\
\hline 14 Testemunha c/ capina & $17,98 \mathrm{a}$ & 8,62 a & $1,75 \mathrm{~b}$ & 0,45 a & $79.688 \mathrm{a}$ \\
\hline 15 Testemunha s/ capina & $0,77 \mathrm{~d}$ & $0,00 \mathrm{c}$ & $0,31 \mathrm{~d}$ & $0,18 \mathrm{c}$ & $0,0 \mathrm{~d}$ \\
\hline $\mathrm{CV}(\%)$ & 29,74 & 41,67 & 23,47 & 20,51 & 32,46 \\
\hline
\end{tabular}

Grupos de médias seguidas da mesma letra, na coluna, não diferem pelo teste Scott-Knott a 5\% de probabilidade.

\section{Conclusões}

1. Aplicações de herbicidas em pré e pósemergência da mandioquinha-salsa reduziram os efeitos da interferência de plantas daninhas com a cultura.
2. As combinações de herbicidas mais promissoras para o controle de plantas daninhas e que permitiram maiores produções de raízes comerciais foram: linuron + fluazifop-p-butil, metolachlor + metribuzin + linuron, diuron + fluazifop-p-butil e atrazine+fluazifop-p-butil. 
3. Os herbicidas atrazine + óleo mineral e oxadiazon, em pós-emergência, proporcionaram sintomas de intoxicação e redução no estande da mandioquinha-salsa.

\section{Referências}

CÂMARA, F. L. Estudo de tecnologias objetivando precocidade de produção de batata-baroa (Arracacia xanthorrhiza Bancroft). 1984. 54p.Dissertação (Mestrado em Fitotecnia) - Universidade Federal de Viçosa, Viçosa: UFV, 1984.

CASALI, V.W.D. Sugestões de adubação para hortaliças Mandioquinha-Salsa. In: RIBEIRO, A. C.; GUIMARÃEs, P.T.G.; ALVARES, V,V.H. Recomendações para uso de corretivos e fertilizantes em Minas Gerais - 5a Aproximação. Viçosa: CFSEMG, 1999. cap.18, p.191.

COOPERATIVE EXTENSION SERVICE AMES. How a soybean plant develops. Ames: Iowa State University of Science and Technology. 1994. 20p.

FREITAS, R.S.; SEDIYAMA, M.A.N.; PEREIRA, P.C.; FERREIRA, F.A.; CECON, P.R.; SEDIYAMA, T. Período de interferência de plantas daninhas na cultura da mandioquinha-salsa. Planta Daninha, Viçosa, v. 22, n. 4, p. 499-506, 2004a.

FREITAS, R.S.; PEREIRA, P.C.; SEDIYAMA, M.A.N.; SEDIYAMA, T.; FERREIRA, F.A. Seletividade de herbicida aplicado em pós-emergência para a cultura da mandioquinhasalsa. Planta Daninha, Viçosa, v. 22, n. 1, p. 159-165, 2004 b.

HENZ, G. P. Doenças da mandioquinha-salsa e sua situação atual no Brasil. Horticultura Brasileira, Brasília, v. 20, n. 2, p. 135-144, 2002.

MASCARENHAS, M.H.T. Controle de plantas daninhas em cenoura e mandioquinha-salsa. Informe Agropecuário, Belo Horizonte, v.10, n.120, p.36-40, 1984.

MASCARENHAS, M.H.T; PEREIRA, W. Manejo de plantas daninhas na cultura da mandioquinha-salsa. Informe Agropecuário, Belo Horizonte, v.19, n.190, p.45-47, 1997.

PEREIRA, P.C.; SEDIYAMA, M.A.N.; FREITAS, R.S; FERREIRA, F.A.; SEDIYAMA, T. Seletividade de herbicidas aplicados em pós-emergência na cultura de mandioquinha-salsa: Revista Ceres, Viçosa, v. 50 , n. 289, p.383-393, 2003.

RODRIGUES, B.N.; ALMEIDA, F.S. Guia de herbicidas. 5.ed. Londrina: Edição dos Autores, 2005. 592p.

SANTOS, F.F. A cultura da mandioquinha-salsa no Brasil. Informe Agropecuário. Belo Horizonte, v.19, n.190, p.5-7, 1997.

SANTOS, F. F.; COSTA, G. P.; MACEDO, P.; KRIECK, R.S. Mandioquinha-salsa no agronegócio do Estado do Paraná. Curitiba: [s.n.], 2000. 56 p. (Informação Técnica, 51)

SEDIYAMA, M.A.N.; CASALI, V.W.D. Propagação vegetativa da mandioquinha-salsa. Informe Agropecuário, Belo Horizonte, v.19, n.190, p.24-27, 1997. 